\title{
Modelling of Newtonian blood flow through a bifurcated artery with the presence of an overlapping stenosis
}

\author{
Norliza Mohd Zain ${ }^{a}$ and Zuhaila Ismail b, ${ }^{*}$ \\ a,b Department of Mathematical Sciences, Faculty of Science, Universiti Teknologi Malaysia, 81310 UTM Johor Bahru, Johor, Malaysia \\ * Corresponding author: zuhaila@utm.my
}

\section{Article history}

Received 23 October 2017

Accepted 8 November 2017

\begin{abstract}
Mathematical modelling of blood flow is developed by treating blood as a Newtonian fluid. In this study, stenosis has been accounted as an overlapping shaped in two-dimensional Cartesian coordinate system. Blood is assumed as steady laminar flow, fully developed and incompressible which flowing through an arterial bifurcation where the wall considered rigid with no-slip condition. Galerkin weighted residual method is performed and a Matlab code is developed to solve the problem. To validate the code, comparison has been made with the results obtained by COMSOL Multiphysics. Results on the velocity profiles and the streamlines pattern are observed and discussed in details.
\end{abstract}

Keywords: finite element method (FEM), Galerkin weighted residual (GWR), newtonian, overlapping stenosis, bifurcated artery

\section{INTRODUCTION}

Numerous studies on the hemodynamic characteristics of blood flow in a stenotic vessels has been performed widely among the researchers not only in a single arterial geometry but also in a bifurcated artery to gain a better understanding on its pathological mechanism. It does not only provide a better insight to the researchers on how blood was distributed through the arteries but it also does helps doctor in the treatment predictions in order to provide a better cure to the patient [1] Partial occlusion in blood vessels or better known as stenosis formed as a results of cholesterols, fats and smooth muscle cells accumulation in the arterial wall [2]. Stenosis is a localized plaque that narrowed the vessel walls causing a major alterations in the flow structure which consequently reduced the fluid flow passing to the other organs and tissues [3]. As the stenosis continues to enlarged and stimulated the development of a blood clot or a "vulnerable plaque", plaque which is prone to rupture, an individual is exposed to the risk of cardiovascular diseases such as stroke and heart attack [1,4-5]. Regions with high curvatures and bifurcations are the most favored sites predisposed to this plaque localization [3]. This preferential localization of atherosclerosis occurred because of the alteration of vessels geometry which involve an immediate variation in the cross sectional area of vessels and the existence of curvature of the artery [6]. Both the vessels geometry and various biomechanical factors influence the pattern of blood flow and might be responsible for the genesis and progression of this disease [7]. The properties of blood is majorly influenced by the hematocrit distribution since red blood cells comprised about $99 \%$ the majority of formed elements suspended in the plasma [8]. Hence, the aggregation and deformation properties of red blood cells dominate the viscous effects of blood as well as its flow. According to Bose and Banerjee [9], in a large diameter artery with shear rates greater than $100 \mathrm{~s}^{-1}$, the representation of blood as a Newtonian fluid is acceptable since the non-Newtonian properties are not significant in this region [10]. Chakravarty and Mandal [11] reported that the blood flow is affected considerably by the overlapping shaped stenosis where the peaks of the velocity distributions was observed at the overlapping region. As far as we know, only a few literatures are concerned on study related to the stenotic artery with an overlapping shaped stenosis [12]. Therefore, a study on a more realistic constrictions like an irregular, overlapping and a multiple stenosis is required.

Motivated by all those studies mentioned, we propose to examine the steady laminar flow through a bifurcated artery with the presence of an overlapping shaped stenosis. The streaming blood is characterised by the Newtonian fluid model. The consideration of blood vessel as a rigid wall is reasonable since the wall of the diseased arteries are less compliant [13]. The solution is obtained numerically by implementing FEM analysis where the discretization of the governing equations is carried out by using Galerkin weighted residual (GWR) method. FEM is chosen instead of any other method since it is a numerical tools that work effectively within arbitrary geometries [14] subject to initial and boundary conditions [13]. Numerical computation will be performed based on the developed algorithms in Matlab software that will be validated using simulations obtained from COMSOL Multiphysics 5.2, a software based on the FEM. In this paper, a detailed evaluation on one of the biomechanical factors that may be responsible for this disease progression which is flow velocity will be performed and discussed in details.

\section{PROBLEM FORMULATION}

In order to formulate the computational domain for the stenosed bifurcated artery, these following assumptions are imposed : 
1. The artery forming bifurcation is of finite length.

2. The parent aorta possesses a single overlapping shaped stenosis in its lumen.

3. Curvatures are introduced at the lateral junctions and the flow divider of the arterial bifurcation to ensure that one can rule out the presence of any discontinuity causing non-existent of separation zones.

\section{Model construction}

The geometry of the stenosed bifurcated artery is modelled as a bifurcated channel as proposed by Chakravarty and Mandal [15]. However, in this study an overlapping shaped stenosis described by Chakravarty and Mandal [11] is considered instead of a mild shaped stenosis as shown in Fig. 1.

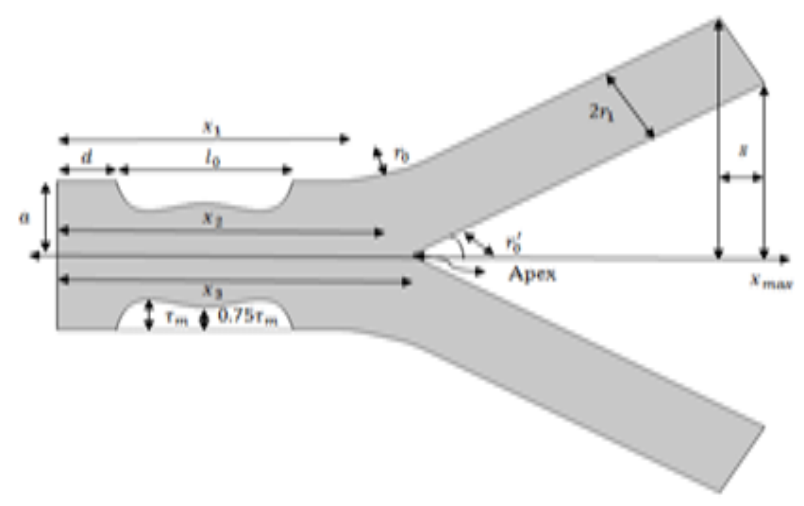

Fig. 1 The geometry of an overlapping stenosis in the bifurcated artery.

Let $(x, y)$ be the coordinates of a material point where the $x$-axis is taken along the axis of the trunk while the $y$-axis is taken along the $y$ direction. The geometry of the bifurcated artery in the presence of an overlapping shaped stenosis may be set up mathematically of which the outer wall geometry is described by

$R_{1}(x)=\left\{\begin{array}{lr}a, & 0 \leq x \leq d \text { and } d+l_{0} \leq x \leq x_{1}, \\ a-\frac{3 \tau_{m}}{2 l_{0}^{4}}\left\{\begin{array}{lr}11(x-d) l_{0}^{3}-47(x-d)^{2} l_{0}^{2} \\ +72(x-d)^{3} l_{0}-36(x-d)^{4}\end{array}\right\}, d \leq x \leq d+l_{0}, \\ a+r_{0}-\sqrt{r_{0}^{2}-\left(x-x_{1}\right)^{2},} & x_{1} \leq x \leq x_{2}, \\ 2 r_{1} \sec \beta+\left(x-x_{2}\right) \tan \beta, & x_{2} \leq x \leq x_{\max }-s .\end{array}\right\}$

On the other hand, the inner wall of geometry can be specified as

$$
R_{2}(x)=\left\{\begin{array}{lc}
0, & 0 \leq x \leq x_{3}, \\
\sqrt{r_{0}^{\prime}-\left(x-\left(x_{3}+r_{0}^{\prime}\right)\right)^{2}}, & x_{3} \leq x \leq x_{4}, \\
r_{0}^{\prime} \cos \beta+\left(x-x_{4}\right) \tan \beta, & x_{4} \leq x \leq x_{\max },
\end{array}\right\}
$$

where $R_{1}(x)$ and $R_{2}(x)$ represent the radii of the outer and inner wall, respectively. Meanwhile, $a$ and $r_{1}$ are the respective radii of the mother and daughter artery. $r_{0}$ and $r_{0}^{\prime}$ are the radii of curvature for the lateral junction and the flow divider, respectively. Whereas, $l_{0}$ is the length of the stenosis at a distance $d$ from the origin. Location of the onset and offset of the lateral junction are denoted by $x_{1}$ and $x_{2}$, respectively. $x_{3}$ indicated as the apex, $\tau_{m}$ represents the maximum height of stenosis occur at $d+l_{0} / 6$ and $d+5 l_{0} / 6$ while $\beta$ denote half of the bifurcation angle. Parameters involved in the above expressions (1) and (2) may be given as

$$
\begin{gathered}
x_{2}=x_{1}+r_{0} \sin \beta, r_{0}=\frac{a-2 r_{1} \sec \beta}{\cos \beta-1}, r_{0}^{\prime}=\frac{\left(x_{3}-x_{2}\right) \sin \beta}{1-\sin \beta}, \\
x_{3}=x_{2}+q, s=2 r_{1} \sin \beta,
\end{gathered}
$$

where $q$ is taken as a small number lying in the range of $0.0001 \leq q \leq 0.0005$ chosen for the compatibility of the geometry and

$$
x_{4}=x_{3}+r_{0}^{\prime}(1-\sin \beta)
$$

\section{Governing equations}

The blood flow in the arterial bifurcation is considered to be in two dimensional, steady, laminar, and incompressible where the rheological characteristics of the flowing blood is characterised by a Newtonian fluid model. Hence, the equations that govern such flow may be written as

$$
\begin{gathered}
\frac{\partial u}{\partial x}+\frac{\partial v}{\partial y}=0, \\
\rho\left[u \frac{\partial u}{\partial x}+v \frac{\partial u}{\partial y}\right]=-\frac{\partial p}{\partial x}+\mu\left(\frac{\partial^{2} u}{\partial x^{2}}+\frac{\partial^{2} u}{\partial y^{2}}\right), \\
\rho\left[u \frac{\partial v}{\partial x}+v \frac{\partial v}{\partial y}\right]=-\frac{\partial p}{\partial y}+\mu\left(\frac{\partial^{2} v}{\partial x^{2}}+\frac{\partial^{2} v}{\partial y^{2}}\right),
\end{gathered}
$$

where $u$ and $v$ represents the velocity of blood in $x$ and $y$ directions, respectively. $\rho$ is the density of blood, $\mu$ is the viscosity of blood and $p$ is the pressure distribution acting on the surface.

\section{Boundary conditions}

At the inlet, a parabolic velocity profile is assumed corresponding to a fully developed flow given by

$$
\begin{gathered}
u(x, y)=u_{\max }\left(1-\frac{y^{2}}{a^{2}}\right) \text { and } v(x, y)=0, \text { at } x=0, \text { and } \\
-a \leq y \leq a .
\end{gathered}
$$

Along all the arterial walls, the usual no-slip conditions are prescribed as

$$
u(x, y)=0, v(x, y)=0 .
$$

At the outlets, a traction-free condition with no tangential and normal forces is applied which can be stated as

$$
(-p \mathbf{I}+\tau) \cdot \mathbf{n}=0
$$

where $\tau$ is the stress tensor and $\mathbf{n}$ represents a unit outward normal vector.

\section{Bubnov-Galerkin weighted residual (GWR) method}

To transform the differential equations, (Eqs. (3)-(5)) into a system of algebraic equations, the spatial discretization of the governing 
equations are performed using the Bubnov-Galerkin weighted residual (GWR) method. These spatial discretization are employed to the interpolate velocity $\mathbf{u}=(u, v)$ and pressure $\mathbf{p}$ by using the unstructured six nodal triangular elements. The spatial variation for the velocity components $u, v$ and the pressure, $p$ are approximated by appropriate quadratic shaped function, $N_{i}(x, y)$ and linear shaped function, $P_{i}(x, y)$, respectively which may be elucidated as follow

$$
u=\sum_{i}^{n} N_{i} u_{i}, v=\sum_{i}^{n} N_{i} v_{i}, p=\sum_{i}^{m} P_{i} p_{i}
$$

where $n=6, m=3$ choosen to represents the number of nodes defined for velocity and pressure components, respectively. The previous definitions (Eqs. (3)-(5)) represent the strong form ( governing differential equations along with natural and essential boundary conditions that must be satisfy at every point over a domain) of the governing equations. To derive the weak form (condition that the solution should satisfy in an integral form with reduced order of derivatives) for the system of governing equations, we utilized the GWR method by weighting the continuity and momentum equations with the appropriate weighting functions, $P_{j}$ and $N_{k}$, respectively. Then, the sum of integration of the resulting equations over each element is equated to zero. Next, integration by part is conducted onto the pressure and viscous terms by applying the product rule for differentiation along with the divergence theorem of Gauss to reduces the differential order. Hence, the system of governing equations (Eqs. (3)-(5)) become as follow

$$
\sum_{1}^{n_{e}}\left[\sum_{j}^{n} \sum_{i}^{n} \int_{A_{e}} P_{j}\left(\frac{\partial N_{i}}{\partial x} u_{i}+\frac{\partial N_{i}}{\partial y} v_{i}\right) d A=0\right],
$$

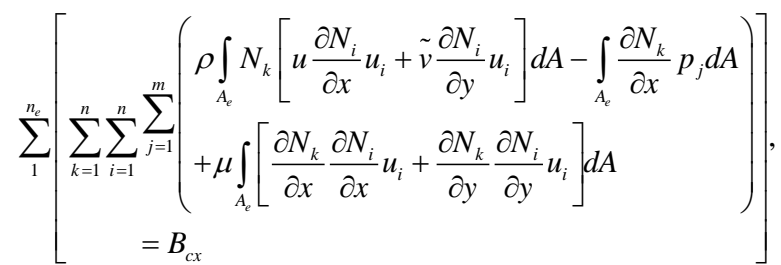

$$
\sum_{1}^{n_{e}}\left[\sum_{k=1}^{n} \sum_{i=1}^{n}\left(\begin{array}{l}
\rho \int_{A_{e}} N_{k}\left[u \frac{\partial N_{i}}{\partial x} v_{i}+\tilde{v} \frac{\partial N_{i}}{\partial y} v_{i}\right] d A-\int_{A_{e}} \frac{\partial N_{k}}{\partial y} p_{j} d A \\
+\mu \int_{A_{e}}\left[\frac{\partial N_{k}}{\partial x} \frac{\partial N_{i}}{\partial x} v_{i}+\frac{\partial N_{k}}{\partial y} \frac{\partial N_{i}}{\partial y} v_{i}\right] d A
\end{array}\right]=B_{c y}\right],
$$

where $j=1,2,3$ and $k=1,2,3,4,5,6$ while $d A=d x d y$. While, $n_{e}$ indicates the total number of elements. On the other hand, the right hand side term for Eqs. (8) and (9) can be clarifed as

$$
\begin{aligned}
& B_{c x}=-\int_{\partial A_{e}} N_{k} P_{j} p_{j} n_{x} d S+\frac{1}{\operatorname{Re}} \int_{\partial A_{e}}\left[N_{k} \frac{\partial N_{i}}{\partial x} u_{i} n_{x}+N_{k} \frac{\partial N_{i}}{\partial y} u_{i} n_{y}\right] d S, \\
& B_{c y}=-\int_{\partial A_{e}} N_{k} P_{j} p_{j} n_{y} d S+\frac{1}{\operatorname{Re}} \int_{\partial A_{e}}\left[N_{k} \frac{\partial N_{i}}{\partial x} v_{i} n_{x}+N_{k} \frac{\partial N_{i}}{\partial y} v_{i} n_{y}\right] d S .
\end{aligned}
$$

The above equations, (Eqs. (7)-(9)) can also be written in the matrix and vector forms which yield

$$
[k]^{\mathrm{e}}\{u\}^{\mathrm{e}}=\{b\}^{\mathrm{e}},
$$

where $[k]^{e}$ represent the local stiffness matrix, while $\{u\}^{\mathrm{e}}$ and $\{b\}^{e}$ respectively termed as the local vector degree of freedom and the local load vector. Note that, $\{b\}^{e}$ are the boundary terms which appeared from the integration by parts procedure as Eq. (10). In an expanded matrix form, matrix (11) can also be represented as

$$
\left[\begin{array}{ccc}
k_{11} & 0 & k_{13} \\
0 & k_{22} & k_{23} \\
k_{31} & k_{32} & 0
\end{array}\right]^{e}\left\{\begin{array}{l}
u_{i} \\
v_{i} \\
p_{j}
\end{array}\right\}^{e}=\left\{\begin{array}{l}
b_{1} \\
b_{2} \\
b_{3}
\end{array}\right\}^{e},
$$

where $u$ and $\tilde{v}$ that appeared in Eqs. (8) and (9) are considered to be constants when the matrices are being formulated. While, for the coeeficient of the vector $b_{1}^{e}, \quad b_{2}^{e}$ and $b_{3}^{e}$ which emerged on the right hand side of matrix (12) due to the neumann boundary conditions imposed and by Eq. (6) they may have these form

$$
\begin{gathered}
b_{1}^{e}=B_{c x}=0, \\
b_{2}^{e}=B_{c y}=0, \\
b_{3}^{e}=0 .
\end{gathered}
$$

To evaluate the integrals, the numerical integration technique called Gaussian quadrature will be adopted after all elements have been assembled forming a global fluid matrix version of equation (12) as follow

$$
[K]\{U\}=\{B\},
$$

where $[K],\{U\}$ and $\{B\}$ are the global stiffness matrix, global vector degree of freedom and global load vector, respectively.

\section{Computational mesh}

Mesh dependency test was performed to ensure the results obtained were not depended on the mesh parameters. Several attempts has been made and only few of them is mentioned here. The number of domain elements computed using COMSOL Multiphysics and Matlab are summarised in Table 1

Table 1 Mesh parameters computed in COMSOL Multiphysics and Matlab.

Software

Parameter

Domain elements

Mesh 1

20153

COMSOL

Multiphysics

Mesh 2

24067

Mesh 3

30896

Mesh 1

15958

Mesh 2

19234

Matlab

Mesh 3

23432

Mesh 4 
Fig. 2 illustrates the axial velocity profiles for various number of mesh evaluated at the center of a mild shaped of stenosis computed using COMSOL Multiphysics and Matlab, respectively.

(a)

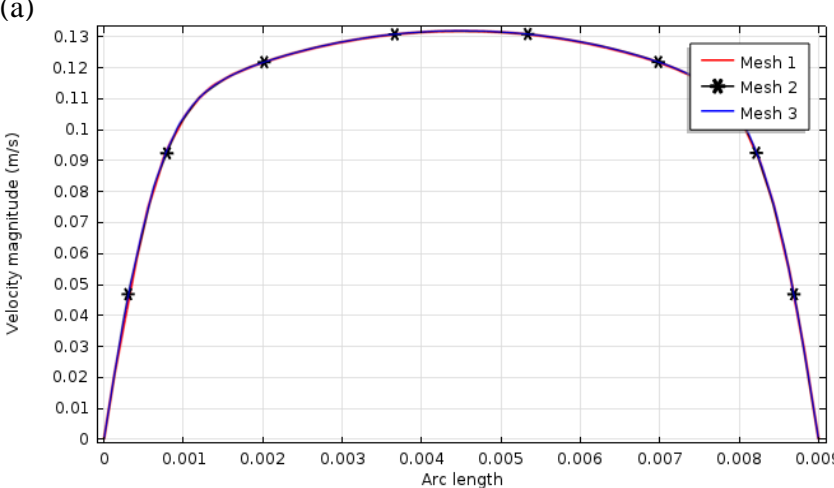

(b)

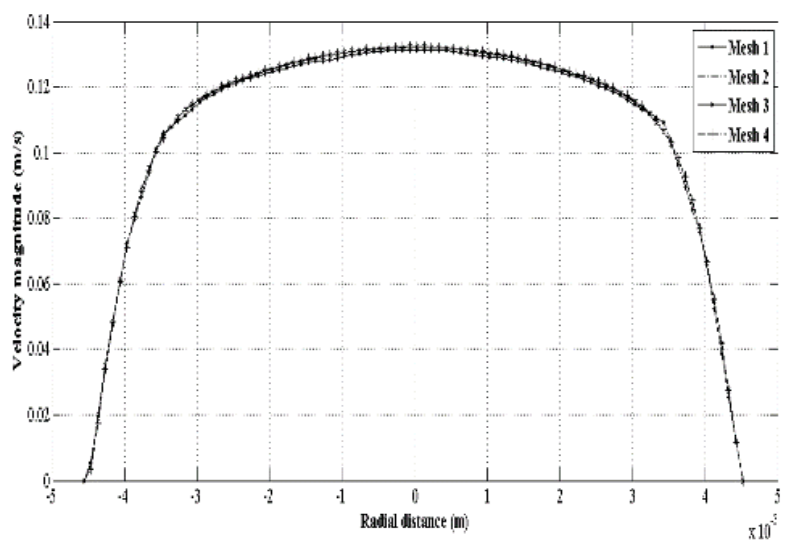

Fig. 2 Axial velocity profiles for different number of domain elements at $x=0.0125 \mathrm{~m}$ evaluated using (a) COMSOL Multiphysics and (b) Matlab

Based on the mesh dependency test demonstrated, to reduce the computational time Mesh 2 computed in COMSOL Multiphysics and Mesh 3 evaluated by Matlab are selected in order to provide a satisfactory solutions to our problem.

\section{Matlab code validation}

The Matlab programming code developed for this numerical computation has been validated with the simulations obtained from COMSOL Multiphysics 5.2 considering problem which possessed a mild shaped stenosis in the bifurcated artery. Fig. 3 shows the comparison on the filled contour plot of velocity obtained from COMSOL Multiphysics and Matlab, respectively with arrows representing the velocity vector.

(a)

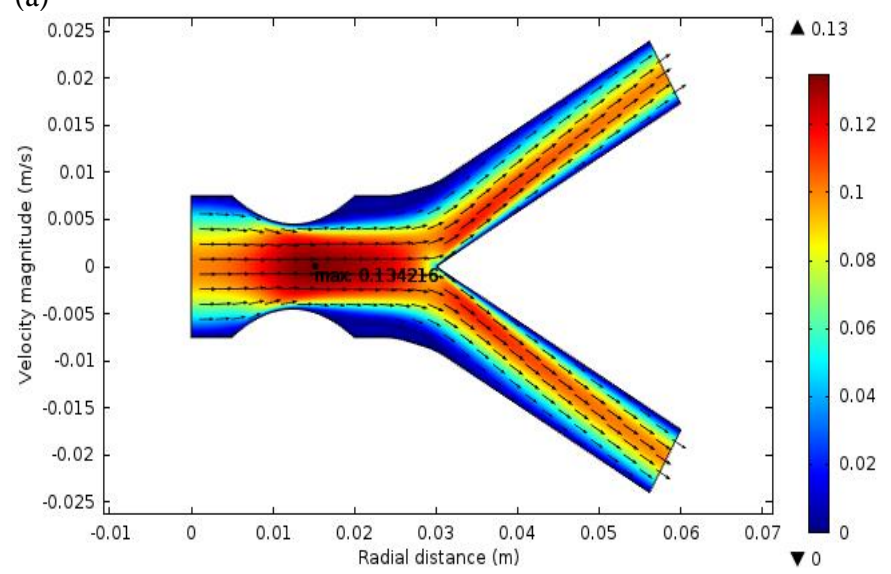

(b)

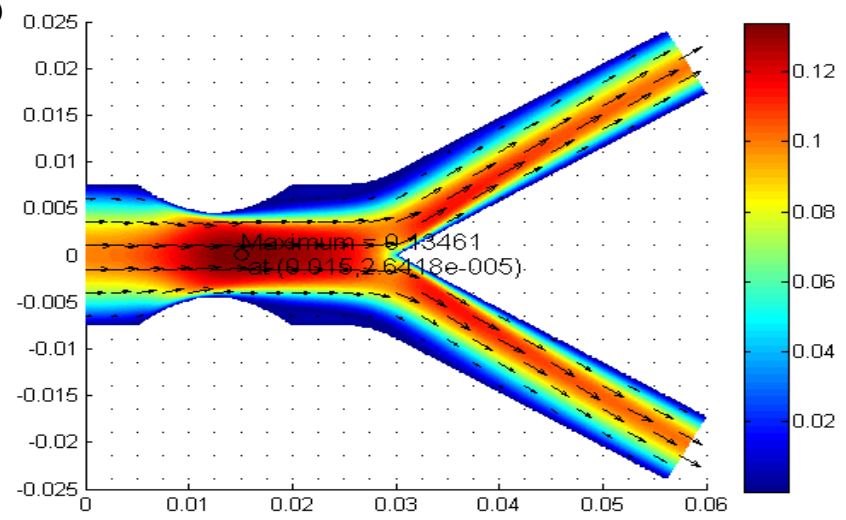

Fig. 3 Filled contour velocity obtained from (a) COMSOL multiphysics and (b) MATLAB computation.

The results obtained from both computations agreed well with each other with a very small difference recorded approximately $0.0004 \mathrm{~m} / \mathrm{s}$ for the maximum velocity. Table 2 consists of the respective maximum velocity obtained from COMSOL Multiphysics and Matlab together with its coordinate.

Table 2 Comparison of maximum velocity and their coordinate.

\begin{tabular}{ccc}
\hline Software & $\begin{array}{c}\text { Maximum } \\
\text { velocity }(\mathbf{m} / \mathbf{s})\end{array}$ & Coordinate $(x, y)$ \\
\hline $\begin{array}{c}\text { COMSOL } \\
\text { Multiphysics }\end{array}$ & 0.134216 & $\left(0.015154,6.9239 \times 10^{-5}\right)$ \\
\hline Matlab & 0.13461 & $\left(0.015,2.6418 \times 10^{-5}\right)$ \\
\hline
\end{tabular}

\section{RESULT AND DISCUSSION}

The numerical computation has been performed in order to estimate the velocity profiles at the mother and daughter artery quantitatively for different severity of an overlapping stenosis. To have a thorough quantitative analysis on the effects of Newtonian blood rheology on the stenotic bifurcated artery flow phenomenon the data have been made use from [3], [15] :

$a=0.0075 \mathrm{~m}, \quad l_{0}=0.015 \mathrm{~m}, d=0.005 \mathrm{~m}, x_{\max }=0.06 \mathrm{~m}, x_{1}=0.025 \mathrm{~m}$, $\rho=1050 \mathrm{kgm}^{-3}, \mu=0.0035 \mathrm{Pas}^{-1}, \beta=30^{\circ}, q=0.0002 \mathrm{~m}, \quad r_{1}=0.51 a$, $\tau_{m}=0.3 a, 0.4 a, 0.5 a$.

\section{Axial velocity profiles for different severity of stenosis}

To further demonstrates the effect of severity of stenosis on the flow of blood, graph on the axial velocity profiles are illustrated as in Fig. 4 (a) and (b) for 30\%, $40 \%$ and 50\% occluded at the second throat of an overlapping stenotic region, $x=0.0175 \mathrm{~m}$ and at the daughter artery, $x=0.04 \mathrm{~m}$, respectively. The percentage of the stenosis is calculated following the formula suggested in [16] as follow

$$
\text { Percentage of severity of stenosis }=\left[1-\left(\frac{D_{\text {stenosis }}}{D_{\text {normal }}}\right)\right] \times 100 \text {, }
$$

where $D_{\text {stenosis }}$ and $D_{\text {normal }}$ represents the diameter of artery at the most severe site and the diameter of the normal healthy artery, respectively. 


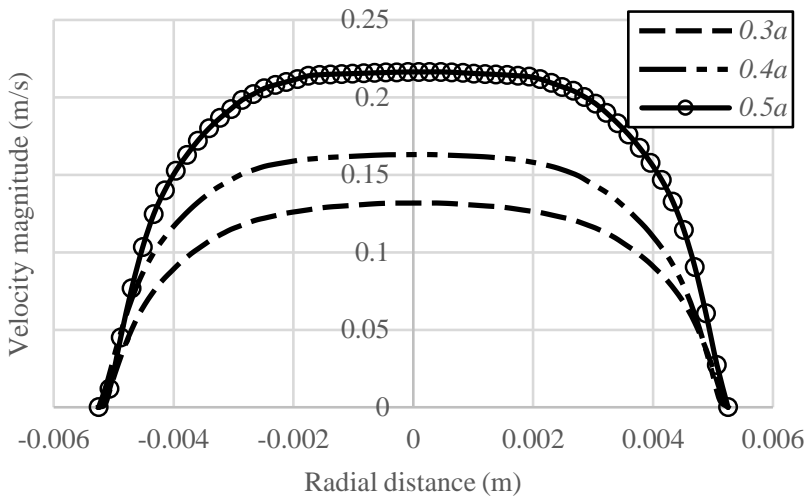

(a)

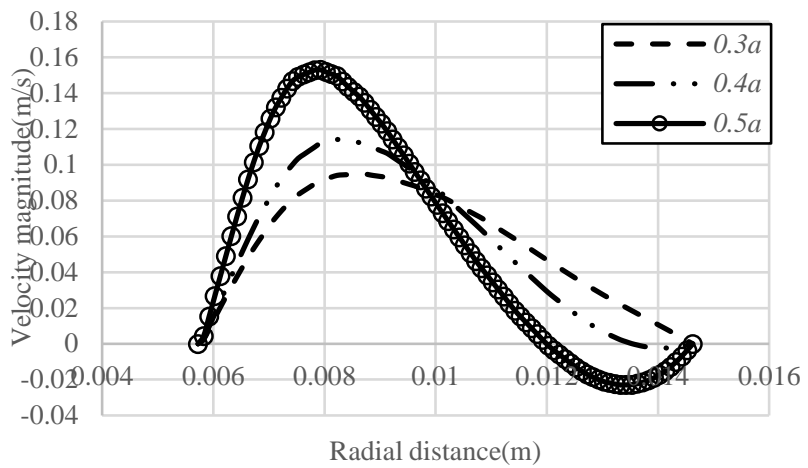

(b)

Fig. 4 Effect of the severity of stenosis on the axial velocity profiles at (a) $x=0.0175 \mathrm{~m}$ and (b) $x=0.04 \mathrm{~m}$.
The observation made in this current figure has shown that an increment on the severity of stenosis enhanced the magnitude of the maximum velocity at the center of the second throat of stenosis. Hence, as the height of the stenosis increases from $\tau_{m}=0.3 a$ to $\tau_{m}=0.4 a$, and $0.5 a$ the peak velocity are found increases substantially from 0.13247 to 0.16416 and 0.21958 , respectively. According to [12], since the area is smaller at the maximum occlusion region hence the velocity increases the most at the center, similar to our findings.

This would later caused to a reduction on velocity magnitude of blood flowing into the daughter artery compared to the velocity recorded on the previous figure as depicted on Fig. 4 (b) for each severity, $\tau_{m}$. This is because some part of the flow had already being decelerated after get obstructed in the mother artery. Moreover, from Fig. 4 (b) also it is clearly observed that, as the stenosis enlarged from $\tau_{m}=0.4 a$ to $0.5 a$, the recirculation areas formed at $x=0.04 \mathrm{~m}$ increases significantly which is indicated by a negative flow near the arterial wall.

\section{Streamlines pattern for different severity of stenosis}

The influence of severity of stenosis on the flow recirculation zones are illustrated on Fig. 5 for severity, $\tau_{m}=0.3 a, 0.4 a, 0.5 \mathrm{a}$. Obviously, the recirculation zones are found to increase in sizes as the stenosis become severe.

In fact, as the severity of stenosis increases to $\tau_{m}=0.4 a$, the formation of vortexes are not only found downstream of the stenotic region but also at the critical height of an overlapping stenosis. As the stenosis height enlarged to $\tau_{m}=0.5 a$, the recirculation area grows even larger at both regions. Hence, the severity of stenosis discovered to enhanced the vortex formation and may lead to a serious complication on the flow characteristics and may also trigger plaque rupture.
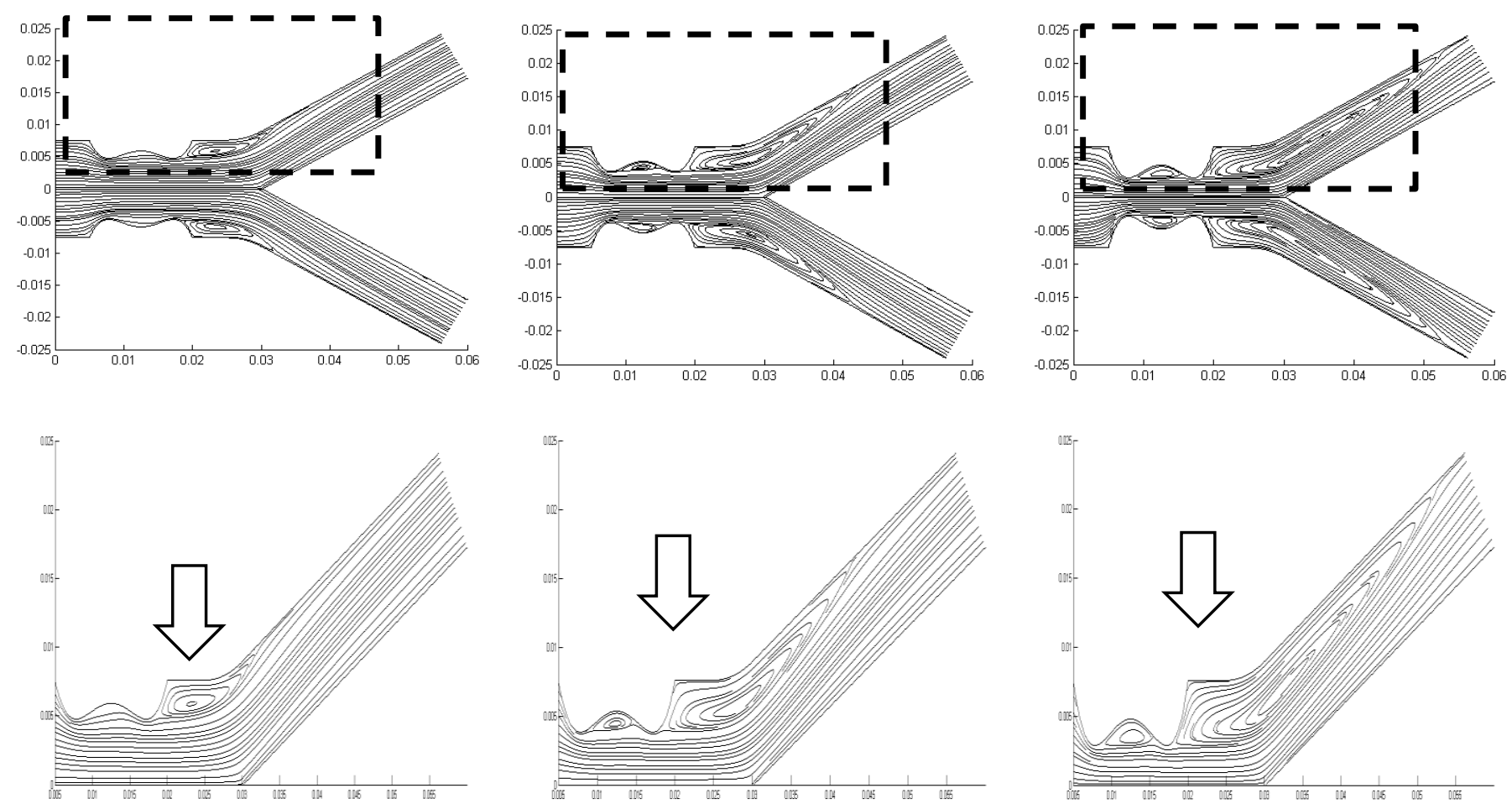

(a)

(b)

(c)

Fig. 5: Effect of severity of stenosis on the recirculation blood zones for (a) $\tau_{m}=0.3 a$, (b) $\tau_{m}=0.4 a$ and (c) $\tau_{m}=0.5 a$. 


\section{CONCLUSION}

A mathematical model of a steady, laminar, incompressible, and Newtonian blood rheology in a stenotic bifurcated artery has been developed where the shape of the stenosis is considered overlapping. The analysis has been carried out on the axial velocity profile at specific region of the mother and daughter artery to investigate the effects of severity of stenosis at various locations. From the outcomes, it is concluded that the increment in the severity of stenosis demonstrates an increment of the velocity profile up to its peak value due to the more slender region of occlusion in the stenotic region which lead to lack of blood flowing through the daughter artery. In addition, as the stenosis grows become more severe, the flow reversal and recirculation zones are formed which might exposed an individual to a worsening effects of cardiovascular diseases. Hence, the overlapping shaped of stenosis does affect the flow velocity of the streaming blood significantly.

The presented research is capable to predict the flow velocity of different degrees of an overlapping shaped stenosis. For future work, a compliant walls can be consider instead of a rigid walls assumption carried out for this current study. Besides that, a non-Newtonian blood rheology should also be taken into account. Study can also incorporates a time and others haemodynamic effects to be discussed in details.

\section{ACKNOWLEDGEMENT}

The authors would like to acknowledge Ministry of Higher Education (MOHE) and Research Management Centre (RMC), Universiti Teknologi Malaysia for the financial support through vote numbers A. J091002.5600.07397, 4F632, 13H28 and 03G53 for this research.

\section{REFERENCES}

[1] Sadek, H.A.A., Khami, M.J. and Obaid, T.A.S. Computer Simulation of Blood Flow in Large Arteries by a Finite Element Method. International Journal of Computer Science and Engineering (IJCSE). 2013. 2(4): 171184.

[2] Zaman, A., Ali, N., Sajid, M., Hayat, T. Effects of Unsteadiness and nonNewtonian Rheology on Blood Flow through a Tapered Time-Variant Stenotic Artery. AIP Advances. 2015. 5(3): 0371291-03712913.
[3] Rabby, M.G., Shupti, S.P., and Molla, M.M. Pulsatile Non-Newtonian Laminar Blood Flows through Arterial Double Stenoses. Journal of Fluids. 2014. 2014(757902): 1-13.

[4] Chaichana, T., Sun, Z., and Jewkes, J. Computational Fluid Dynamics Analysis of the Effect of Plaques in the Left Coronary Artery. Computational and Mathematical Methods in Medicine. 2012. 2012(504367): 1-9.

[5] Ismail, Z., Abdullah, I., Mustapha, N., and Amin, S. A Power-Law Model of Blood Flow through a Tapered Overlapping Stenosed Artery. Applied Mathematics and Computation. 2008. 195(2): 669-680.

[6] Ryou, H.S., and Ro, K.C. Numerical Study on Turbulent Blood Flow in a Stenosed Artery Bifurcation under Periodic Body Acceleration using a Modified k- $\varepsilon$ Model. Korea-Australia Rheology Journal. 2010. 22(2): 129139.

[7] Liu, B., and Tang, D. Influence of non-Newtonian Properties of Blood on Wall Shear Stress in Human Atherosclerotic Right Coronary Arteries. Mol Cell Biomech. 2011. 8(1): 73-90.

[8] Sherwood, J.M., Jonathan, D., and Balabani, S. Hematocrit, Viscosity and Velocity Distributions of Aggregating and non-Aggregating Blood in a Bifurcating Microchannel. 2012. Springer. 13(2014): 259-273.

[9] Bose, S., and Banerjee, M. Magnetic Particle Capture for Biomagnetic Fluid Flow in Stenosed Aortic Bifurcation Considering Particle-Fluid Coupling. Journal of Magnetism and Magnetic Materials. 2015. 385(2015): 32-46.

[10] Alimohamadi, H., Imani, M., and Forouzandeh, B. Computational Analysis of Transient non-Newtonian Blood Flow in Magnetic Targeting Drug Delivery in Stenosed Carotid Bifurcation Artery. International Journal of Fluid Mechanics Research. 2015. 42(2): 149-169.

[11] Chakravarty, S., and Mandal, P.K. Mathematical Modelling of Blood Flow through an Overlapping Arterial Stenosis. Mathematical and Computer Modelling. 1994. 19(1): 59-70.

[12] Srivastava, V., Vishnoi, R., Singh, D., Medhavi, A., and Mishra, S. Response to Blood Flow through a Overlapping Stenosis in Catheterized Arteries. e-Journal of Science \& Technology. 2011. 6(5): 93-103.

[13] Sousa, L., Castro, C., Antonio, C., and Chaves, R. Computational Techniques and Validation of Blood Flow Simulation. Wseas Transactions on Biology and Biomedicine. 2011. 8(4): 145-156.

[14] Raptis, A., Xenos, M., Tzirtzilakis, E., and Matsagkas, M. Finite Element Analysis of Magnetohydrodynamic Effects on Blood Flow in an Aneurysmal Geometry. Physics of Fluids. 2014. 26(10): 101901-101914.

[15] Chakravarty, S., and Mandal, P.K. An Analysis of Pulsatile Flow in a Model Aortic Bifurcation, International Journal of Engineering Science. 1997. 35(4): 409-422.

[16] Schwartz, J. Calculating Percent Stenosis, American Journal of Neuroradiology. 2001. 22(1): 228-228. 